\title{
The Banzhaf Value in the Presence of Externalities
}

\author{
M. Álvarez-Mozos ${ }^{1}$ and O. Tejada*2 \\ ${ }^{1}$ Department of Economic, Financial, and Actuarial Mathematics \\ University of Barcelona, Spain. \\ ${ }^{2}$ CER-ETH Center of Economic Research, ETH Zurich, Switzerland.
}

\begin{abstract}
We propose two generalizations of the Banzhaf value for partition function form games. In both cases our approach is based on probability distributions over the set of coalition structures that may arise for any given set of players. First, we introduce a family of values, one for each collection of these latter probability distributions, defined as the Banzhaf value of a coalitional game obtained as the expectation taken according to the given probability distributions of the original partition function form game. For each value of the family we provide two characterization results within the set of all partition function form games. Both results rely on a property of neutrality with respect to the amalgamation of players. Second, we propose another family of values that differ from the previous ones in that the latter values take into account only the information about the most likely coalition structure that may arise according to the given probability distributions. Each value of the second family is also characterized in two results by means of a collusion neutrality property. Unlike the characterizations of the first approach, these characterizations can be restricted to the set of simple games in partition function form.

Keywords: Banzhaf value; Externalities; Games in Partition Function Form; Simple Games
\end{abstract}

\section{Introduction}

One of the most fruitful applications of game theory to the political sciences is the study of power in decision making bodies. Generally power is understood as the ability of an agent to affect the outcome of a voting procedure. A number of power indices have been proposed in the literature to quantify this ability. The most relevant of these measures are the Shapley-Shubik index (Shapley and Shubik, 1954) and the Banzhaf index (Banzhaf, 1964). Both of them build

\footnotetext{
${ }^{*}$ Corresponding author. E-mail address: toriol@ethz.ch
} 
on a common underlying assumption: members of a legislature (be they individuals or parties) may form coalitions, and a coalition is winning if and only if the number of votes cast by its members reaches an exogenously given threshold (most often the simple majority). Accordingly, the bargaining possibilities of the members of a legislature are described by a simple coalitional game, which assigns 1 to winning coalitions and 0 to the rest.

Nevertheless, quite often there are voting procedures in legislatures for which endogenous majority rules are used instead of fixed majority rules. For instance, when the proclamation of the president is a duty of the parliament and no candidate receives the support of at least half of the legislature, governments backed by only a minority of the legislators may be formed. ${ }^{1}$ In these circumstances, the minority government comes to power because the parties that do not back it cannot agree to vote in favor of an alternative candidate. When endogenous majority voting rules apply the bargaining possibilities of the parties cannot be fully captured by a coalitional game. The reason for this is the following: whether a coalition of parties is winning or not is not only determined by how many seats the coalition has but also by the coalition strategies of the remaining parties.

To take situations like the previous one into consideration, Thrall and Lucas (1963) devised the so-called partition function form games (or games in partition function form). In a partition function form game the worth of a coalition depends on how the players outside the coalition are organized, hence allowing for externalities to be incorporated into the framework of coalitional games. Some years later Myerson (1977) proposed and characterized an extension of the Shapley value for partition function form games. It has only been recently, however, that these games have gained importance and some further relevant contributions have been made. For instance, Hafalir (2007) has studied the core of coalitional games with externalities and obtained the necessary conditions for their non-emptiness. The problem of extending the Shapley value to games in partition function form has attracted much attention and several alternatives to Myerson (1977) have since been proposed. First, Macho-Stadler et al. (2007) have characterized a value that can be obtained as the Shapley payoffs of a certain coalition game. Second, de Clippel and Serrano (2008) have followed an axiomatic approach to single out a value that is not affected by externalities. Third, Dutta et al. (2010) have provided a family of values using the notion of potential and have characterized it by means of a reduced game property (see Hart and MasColell, 1989). Other recent contributions include Albizuri et al. (2005) and Pham Do and Norde (2007). To the best of our knowledge, no extension of the Banzhaf value has yet been proposed within the framework of all games in partition function form.

The Banzhaf value was first proposed for voting games (Banzhaf, 1964; Penrose, 1946) and later extended to the class of all coalitional games by Owen (1975). As for the Shapley value, marginal contributions of players to coalitions are the basis of the Banzhaf value. However, while

\footnotetext{
${ }^{1}$ For instance, this has recently been the case in the formation process of the regional governments in the Basque Country and Catalonia.
} 
the Shapley value considers orderings of players to be equally likely, the Banzhaf value considers coalitions of players to be equally likely. From an axiomatic perspective, the main difference is that the Shapley value is efficient while the Banzhaf value satisfies interesting amalgamation neutrality properties.

In the first part of the paper, we propose a family of values for arbitrary games in partition function form. Each value in the family is calculated by applying the Banzhaf value to a coalitional game obtained as the expected average of the original partition function form game taken according to some given probability distributions over the set of possible coalition structures that may arise for any given set of players. Then, by means of an amalgamation neutrality property we provide two characterization results for each value in the family.

In the second part of the paper, we propose an alternative family of values that generalize the Banzhaf value when externalities are present. This second family of values can be considered an ordinal counterpart of the first family in the following sense: even though the latter values are also based on probability distributions over coalition structures, they only take into account the most likely coalition structure for each given set, so they disregard the remaining information included in the probability distributions. By so doing, we define values that fit situations where it may not be possible to assess the exact probability of emergence of every coalition structure but it is possible to identify which the most likely coalition structure is. Each of the values in the family is then characterized using a modification of the previous amalgamation neutrality property. Unlike in the case of the first family of values, the characterization results of the values of the second family remain valid when restricted to the subclass of simple games in partition function form. Both families of values coincide when there are no externalities, as in this case each of their members reduces to the Banzhaf value for coalitional games.

We note that in the framework of simple games with externalities different extensions of the Banzhaf value have been studied to date. In a series of papers, Bolger (1983, 1986, 1990) studied different generalizations of the Banzhaf value for multi-candidate voting games. These latter games are particular instances of simple games in partition function form. Further, Bolger (1990) characterized one of his proposals within the class of values that can be built as weighted averages of marginal contributions, for a certain family of weights. Although the values we study here do not lie within the class of values considered in Bolger (1990), they do satisfy the dummy independence property of his characterization. Simple games in partition function form as considered here belong to the wider class of games with $r$-alternatives (see Freixas and Zwicker, 2003). The latter is a model that allows for different levels of approval; that is, besides knowing which coalitions are formed, what each of the coalitions is voting for is also known. Bolger (2002) proposed and characterized an extension of the Banzhaf value for games with $r$-alternatives.

As already mentioned, the main results of this paper concern the characterization of values by means of amalgamation neutrality properties that describe how the payoffs of two players 
should change when they decide to act as a single entity. To the best of our knowledge, Lehrer (1988) was the first to consider a property of this kind, which requires that the joint payoff of two players in a game be at most the payoff obtained when they act together as a new player in a reduced game. Haller (1994) used an amalgamation neutrality property that had two important differences with respect to Lehrer's: first, the player set does not change but instead one of the two players becomes null; second, the payoffs in the two situations are required to be equal. Further, Nowak (1997) used another property in which the player set changes and the payoffs are required to be equal. In Malawski (2002) several properties based on Haller's collusion neutrality property were studied and compared. Very recently, Casajus (2012) has used an amalgamation neutrality property in line with the one used by Nowak (1997). ${ }^{2}$ In common with Casajus (2012), the properties that we use here assume that the player set changes due to the exit of one of the merged players and require the equality of payoffs. Nevertheless, important differences arise as we deal with games with externalities and we consider that the two-player amalgamation agreement is asymmetric in the following sense: one player agrees to delegate her role to another player (who acts as her representative) in order to produce some value within an "active" coalition; when the coalition considered is not "active", however, we assume that the agreement is not enforceable and hence the latter player no longer acts necessarily as the representative of the former. ${ }^{3}$

The rest of the paper is organized as follows. In Section 2 we present the first family of values for games in partition function form. In Section 3 we prove two characterization results for each member of the family. In Section 4 we turn our interest primarily to simple games in partition function form, and we present the second family of values for games in partition function form and characterize each of its members in two different results. Section 5 concludes.

\section{A Banzhaf-based Value}

Let $\Omega$ be a (possibly infinite) set of potential players. ${ }^{4}$ Throughout the paper, we let $N \subseteq \Omega$ denote a non-empty finite set of players in $\Omega$, and we indicate the cardinality of $N$ by $n$. A partition (or coalition structure) of $N$, denoted by $P$, is a division of $N$ into pairwise disjoint coalitions, i.e., $P \subseteq\{S: S \subseteq N\}$ such that $\cup_{S \in P}=N$ and for every $S, T \in P$ with $S \neq T$, $S \cap T=\emptyset$. For convenience, we assume that the empty set is an element of every partition. We denote by $\mathcal{P}(N)$ the set of all partitions of $N$. An embedded coalition of $N$ is a pair $(S, P)$ where $P \in \mathcal{P}(N)$ and $S \in P$. The set of embedded coalitions of $N$ is $E C^{N}=\{(S, P): P \in$ $\mathcal{P}(N)$ and $S \in P\}$. Given $S \subseteq N$ and $i \in N, S_{-i}$ (resp. $S_{+i}$ ) stands for the set $S \backslash\{i\}$ (resp. $S \cup\{i\})$. Similarly, given $P \in \mathcal{P}(N)$ and a nonempty coalition $S \in P$, we let $P_{-S} \in \mathcal{P}(N \backslash S)$

\footnotetext{
${ }^{2}$ The difference between these two properties has been studied in Alonso-Meijide et al. (2012).

${ }^{3}$ The asymmetry in the delegation agreement is discussed in more detail in Section 3.

${ }^{4}$ We restrict our attention to non-singleton universes of players.
} 
denote the partition $P \backslash\{S\}$. Lastly, given $P \in \mathcal{P}(N)$ and an arbitrary coalition $T \subseteq N$, $P \backslash T \in \mathcal{P}(N \backslash T)$ stands for the partition obtained from $P$ by removing all players that belong to $T$, i.e., $P \backslash T=\{R \backslash T: R \in P\}$.

A game in partition function form (or simply a game) is a pair $(N, v)$, where $N \subseteq \Omega$ and $v$ is a function that assigns to every embedded coalition its "worth", i.e., $v: E C^{N} \rightarrow \mathbb{R}$, with the convention that for every $P \in \mathcal{P}(N), v(\emptyset, P)=0$. The set of all games in partition function form with common player set $N$ is denoted by $\mathcal{G}^{N}$. The set of games in partition function form with an arbitrary set of players is denoted by $\mathcal{G}$, i.e., $\mathcal{G}=\bigcup_{\emptyset \neq N \subseteq \Omega} \mathcal{G}^{N}$. For each $N \subseteq \Omega, \mathcal{G}^{N}$ is a vector space. Given $(S, P) \in E C^{N}$, with $S \neq \emptyset$, let $\left(N, e_{(S, P)}\right) \in \mathcal{G}$ be the game defined for every $(T, Q) \in E C^{N}$ by

$$
e_{(S, P)}(T, Q)= \begin{cases}1 & \text { if } S \subseteq T \text { and } \forall T^{\prime} \in Q_{-T}, \exists S^{\prime} \in P \text { such that } T^{\prime} \subseteq S^{\prime} \\ 0 & \text { otherwise. }\end{cases}
$$

From de Clippel and Serrano (2008) we know that $\left\{\left(N, e_{(S, P)}\right):(S, P) \in E C^{N}\right.$ and $\left.S \neq \emptyset\right\}$ constitutes a basis of $\mathcal{G}^{N}$.

For convenience, we consider coalitional games (without externalities) as a subclass of games in partition function form. Formally, $(N, v) \in \mathcal{G}$ is a coalitional game if for every $S \subseteq N$ and $\left(S, P_{1}\right),\left(S, P_{2}\right) \in E C^{N}$,

$$
v\left(S, P_{1}\right)=v\left(S, P_{2}\right) .
$$

In the case of coalitional games, we may simply denote by $v(S)$ the worth obtained by any coalition $S \subseteq N$, as it is independent of how the remaining players organize themselves. The set of all coalitional games with common player set $N \subseteq \Omega$ is denoted by $\mathcal{C G}^{N}$. If we let $\mathcal{C G}=\bigcup_{\emptyset \neq N \subseteq \Omega} \mathcal{C G}^{N}$, we have $\mathcal{C G} \subseteq \mathcal{G}$. A game $(N, v) \in \mathcal{C G}$ is a simple coalitional game if $v(S) \in\{0,1\}$ for all $S \subseteq N, v(N)=1$, and $v(S) \leq v(T)$ for all $S, T \subseteq N$ with $S \subseteq T{ }^{5}$

Lastly, given $\mathcal{H} \subseteq \mathcal{G}$, a value on $\mathcal{H}$ is any mapping, $\mathrm{f}$, that assigns to every game $(N, v) \in \mathcal{H}$ a vector $\mathrm{f}(N, v) \in \mathbb{R}^{N}$ with coordinates $\mathrm{f}_{i}(N, v)$ for all $i \in N$.

\subsection{A family of values}

In the following we define a new family of values for games in partition function form. To do so we require further elements. For each $N \subseteq \Omega$, let $\lambda^{N}: E C^{N} \rightarrow \mathbb{R}_{+}$be a mapping such that for every $S \subseteq N,{ }^{6}$

$$
\sum_{P \in \mathcal{P}(N): S \in P} \lambda^{N}(S, P)=1
$$

That is, $\lambda^{N}$ yields, for every coalition, $S \subseteq N$, a probability distribution over the set $E C^{N, S}=$ $\left\{(S, P) \in E C^{N}\right\}$. Alternatively we can interpret that $\lambda^{N}$ contains the frequencies with which

\footnotetext{
${ }^{5}$ We point out that there are some authors (e.g. Casajus (2012)) that use "simple games" to refer to a class of coalitional games where no monotonicity condition is imposed.

${ }^{6}$ We note that $\mathbb{R}_{+}=[0, \infty)$.
} 
different coalition structures arise. This second interpretation has a special appeal when we consider the example of a legislature as outlined in the Introduction. Indeed, the coalitions formed in a parliament between parties vary depending on the bill being voted, and hence $\lambda^{N}$ can summarize the average behavior of parties in terms of voting alliances.

Throughout the paper, we denote by $\Lambda=\left\{\lambda^{N}: N \subseteq \Omega\right\}$ any set of collections of probability distributions, one for each possible player set $N \subseteq \Omega$. Additionally, unless stated to the contrary we henceforth also assume that the probability distributions satisfy the following two requirements. ${ }^{7}$

a) For every $N, N^{\prime} \subseteq \Omega,(S, P) \in E C^{N}$, and $\left(S^{\prime}, P^{\prime}\right) \in E C^{N^{\prime}}$ such that $P_{-S}=P_{-S^{\prime}}^{\prime}$,

$$
\lambda^{N}(S, P)=\lambda^{N^{\prime}}\left(S^{\prime}, P^{\prime}\right) .
$$

b) $\Lambda$ is consistent, i.e., for every $N \subseteq \Omega, j \in N$, and $(S, P) \in E C^{N_{-j}}$,

$$
\lambda^{N_{-j}}(S, P)=\sum_{T \in P_{-S}} \lambda^{N}\left(S, P_{-T} \cup\left\{T_{+j}\right\}\right) .
$$

First, a) requires that the probability distribution over $E C^{N, S}$ depends only on the set of players not in $S$. This is equivalent to assuming that there exists a family of functions $\left\{\mu^{T}: \mathcal{P}(T) \longrightarrow \mathbb{R}_{+}\right\}_{T \subseteq \Omega}$ such that $\lambda^{N}(S, P)=\mu^{N \backslash S}\left(P_{-S}\right)$ for all $(S, P) \in E C^{N}$. The idea behind this requirement is thus to assume that there are exogenously given beliefs about how any set of players organizes itself into a coalition structure independently of the players outside the set.

Second, $\Lambda$ is consistent if given some $j \in N, S \subseteq N_{-j}$, and $(S, P) \in E C^{N_{-j}}$, the probability that players in $N_{-j} \backslash S$ are organized according to $P_{-S}$ is independent of whether we consider $j$ or not. ${ }^{8}$ Lastly, we use $\mathcal{L}$ to denote the set of $\Lambda$ that satisfy conditions a) and $\mathbf{b}$ ). We stress that there exist infinitely many such $\Lambda$.

Example 2.1. In this example we introduce some beliefs. For notational convenience, for $N \subseteq$ $\Omega$ and $S \subseteq N$, let $(S, *) \in E C^{N}$ and $(S, * *) \in E C^{N}$ be such that $*=\{\emptyset, S, N \backslash S\} \in \mathcal{P}(N)$ and $* *=\left\{\emptyset, S,\{i\}_{i \in N \backslash S}\right\} \in \mathcal{P}(N)$. Define $\Lambda^{*}$ to be such that for every $N \subseteq \Omega$ and $(S, P) \in E C^{N}$,

$$
\lambda^{N}(S, P)= \begin{cases}1 & \text { if }(S, P)=(S, *), \\ 0 & \text { otherwise. }\end{cases}
$$

Similarly, let $\Lambda^{* *}$ be such that for every $N \subseteq \Omega$ and $(S, P) \in E C^{N}$,

$$
\lambda^{N}(S, P)= \begin{cases}1 & \text { if }(S, P)=(S, * *), \\ 0 & \text { otherwise. }\end{cases}
$$

\footnotetext{
${ }^{7}$ In Section 4 we do not impose the consistency requirement given by condition $\mathbf{b}$ ).

${ }^{8} \mathrm{We}$ opt for consistency, as the property considered relates the probability that players in $N_{-j} \backslash S$ are organized according to $P_{-S}$ within two different nested scenarios: one where $j$ is present, one where $j$ is absent.
} 
Furthermore, given $p \in[0,1]$ define $\Lambda^{p}$ to be such that for every $N \subseteq \Omega$ and $(S, P) \in E C^{N}$,

$$
\lambda^{N}(S, P)= \begin{cases}p & \text { if }(S, P)=(S, *), \\ 1-p & \text { if }(S, P)=(S, * *), \\ 0 & \text { otherwise. }\end{cases}
$$

when $|N \backslash S|>1$, and $\lambda^{N}(S, P)=1$ when $S=N_{-k}$ for some $k \in N$. Trivially, $\Lambda^{*}=\Lambda^{1}$ and $\Lambda^{* *}=\Lambda^{0}$. Moreover, it is straightforward to see that $\Lambda^{p} \in \mathcal{L}$ for all $p \in[0,1]$.

We point out that there exist other, more sophisticated $\Lambda$ satisfying conditions a) and $\mathbf{b}$ ).

Example 2.2. In this example we introduce some other beliefs. Let $\Omega=\Omega^{+} \cup \Omega^{-}$, with $\Omega^{+} \cap \Omega^{-}=\emptyset$ and $N \subseteq \Omega$. For $S \subseteq N$, let $S^{+}=S \cap \Omega^{+}$and $S^{-}=S \cap \Omega^{-}$, and define $\hat{\Lambda}$ to be such that for every $N \subseteq \Omega$ and $(S, P) \in E C^{N}$,

$$
\lambda^{N}(S, P)= \begin{cases}1 & \text { if }(N \backslash S)^{+} \in P \text { and }\{k\} \in P \text { for each } k \in(N \backslash S)^{-}, \\ 0 & \text { otherwise. }\end{cases}
$$

It can be checked that $\hat{\Lambda} \in \mathcal{L}$.

Following the approach used in Macho-Stadler et al. (2007), given $\Lambda \in \mathcal{L}, N \subseteq \Omega$ and $(N, v) \in \mathcal{G}^{N}$, we can associate a number with any coalition $S \subseteq N$ by considering the expected worth of coalition $S$ in $(N, v)$ according to $\lambda^{N} \in \Lambda$, i.e. ${ }^{9}$

$$
v^{\Lambda}(S)=\sum_{P \in \mathcal{P}(N): S \in P} \lambda^{N}(S, P) \cdot v(S, P) .
$$

The coalitional game $\left(N, v^{\Lambda}\right)$ thus averages the externalities on the worth of a given coalition imposed by the outside players via the coalition structure in which they potentially organize.

We are now in a position to introduce the first family of values, one for every $\Lambda \in \mathcal{L}$.

Definition 2.1. Given $\Lambda \in \mathcal{L}$, the $\Lambda$-Banzhaf value, $\mathrm{Ba}^{\Lambda}$, is the value defined for every $(N, v) \in$ $\mathcal{G}$ and $i \in N$ by

$$
\operatorname{Ba}_{i}^{\Lambda}(N, v)=\frac{1}{2^{n-1}} \sum_{S \subseteq N_{-i}}\left[v^{\Lambda}\left(S_{+i}\right)-v^{\Lambda}(S)\right]
$$

The values in (3) are built by means of a two-step procedure. First, the beliefs obtained from $\Lambda$ are used to define a weighted average coalitional game, namely $\left(N, v^{\Lambda}\right)$. Second, the Banzhaf value is applied to the latter coalitional game. Even though our approach in the first step is very similar to that adopted by Macho-Stadler et al. (2007), our assumptions regarding the weights differ from those considered therein. An important reason for this is that we work with variable player sets, as the next section will make evident. Moreover, we do not assume that the weights are independent of the identity of the players. ${ }^{10}$

\footnotetext{
${ }^{9}$ Note that we actually associate a coalitional game with every game in partition function form.

${ }^{10}$ See Example 2.2.
} 


\section{Properties and Characterizations of the $\Lambda$-Banzhaf Value}

In this section we do two things. First, we present and discuss some properties that a value for games in partition function form may satisfy. Second, we present two characterization results for the $\Lambda$-Banzhaf value in the framework of all partition function form games.

\subsection{Some properties}

In order to introduce the first two properties we consider an adaptation to our setting of the standard dummy player property for coalitional games. Given $\Lambda \in \mathcal{L}$ and $N \subseteq \Omega$, we say that $i \in N$ is a $\Lambda$-dummy player in $(N, v) \in \mathcal{G}$ if for every $S \subseteq N_{-i}$,

$$
v^{\Lambda}\left(S_{+i}\right)-v^{\Lambda}(S)=v^{\Lambda}(\{i\}),
$$

or, equivalently,

$$
\begin{aligned}
& \sum_{P \in \mathcal{P}(N): S_{+i} \in P} \lambda^{N}\left(S_{+i}, P\right) \cdot v\left(S_{+i}, P\right)-\sum_{P \in \mathcal{P}(N): S \in P} \lambda^{N}(S, P) \cdot v(S, P) \\
= & \sum_{P \in \mathcal{P}(N):\{i\} \in P} \lambda^{N}(\{i\}, P) \cdot v(\{i\}, P) .
\end{aligned}
$$

That is, $i$ is a $\Lambda$-dummy player in $(N, v) \in \mathcal{G}$ if the value she expects to add to any coalition is, according to the probability distributions given by $\Lambda$, equal to the value she expects to obtain when she remains a singleton. Note that, for an appropriately chosen $\Lambda$, a player may be a $\Lambda$-dummy player in a game even if all her marginal contributions differ. The mild condition in the definition of the dummy player makes to some extent, the $\Lambda$-dummy property below highly demanding.

$\Lambda$-DPP Given $\Lambda \in \mathcal{L}$, a value on $\mathcal{G}, \mathrm{f}$, satisfies the $\Lambda$-dummy player property if for every $N \subseteq \Omega$ and $(N, v) \in \mathcal{G}$ such that $i \in N$ is a $\Lambda$-dummy player in $(N, v)$,

$$
\mathrm{f}_{i}(N, v)=v^{\Lambda}(\{i\}) .
$$

When there are no externalities, i.e. $(N, v) \in \mathcal{C G}$, if a player $i \in N$ is a $\Lambda$-dummy player in $(N, v)$ for some $\Lambda \in \mathcal{L}$ then she is so for every $\Lambda^{\prime} \in \mathcal{L}$. In such a situation we simply say that $i$ is a dummy player in $(N, v)$ with no reference to $\Lambda$. It can be easily checked that when there are no externalities, $\Lambda$-DPP implies the 2-Efficiency property for coalitional games of Casajus (2012). As noted above $\Lambda$-DPP constitutes a strong property. As a consequence, we next introduce a weaker version of $\Lambda$-DPP, obtained from its being required only for games in $\mathcal{C G}$ so that it becomes the standard dummy player property for coalitional games.

$\operatorname{DPP}(\mathrm{w})$ A value on $\mathcal{G}, \mathrm{f}$, satisfies the dummy player property in the weak sense if for every $N \subseteq \Omega,(N, v) \in \mathcal{C G}$ such that $i \in N$ is a dummy player in $(N, v)$,

$$
\mathrm{f}_{i}(N, v)=v(\{i\}) .
$$


Next, we introduce a property that considers a certain merging of players. Given $\Lambda \in \mathcal{L}$, $N \subseteq \Omega, i, j \in N$, with $i \neq j$, and $(N, v) \in \mathcal{G}^{N}$, the $\{i j\}$-reduced game, which we denote by $\left(N_{-j}, v_{(\Lambda, i j)}\right) \in \mathcal{G}^{N_{-j}}$, is defined for every $(S, P) \in E C^{N_{-j}}$ by ${ }^{11}$

$$
v_{(\Lambda, i j)}(S, P)= \begin{cases}v\left(S_{+j}, P_{-S} \cup\left\{S_{+j}\right\}\right) & \text { if } i \in S, \\ \frac{\sum_{T \in P_{-S}} \lambda^{N}\left(S, P_{-T} \cup\left\{T_{+j}\right\}\right) \cdot v\left(S, P_{-T} \cup\left\{T_{+j}\right\}\right)}{\sum_{T \in P_{-S}} \lambda^{N}\left(S, P_{-T} \cup\left\{T_{+j}\right\}\right)} & \text { if } i \notin S,\end{cases}
$$

if $\sum_{T \in P_{-S}} \lambda^{N}\left(S, P_{-T} \cup\left\{T_{+j}\right\}\right)>0$ and

$$
v_{(\Lambda, i j)}(S, P)= \begin{cases}v\left(S_{+j}, P_{-S} \cup\left\{S_{+j}\right\}\right) & \text { if } i \in S, \\ 0 & \text { if } i \notin S,\end{cases}
$$

otherwise. That is, $\left(N_{-j}, v_{(\Lambda, i j)}\right)$ is the resulting game in partition function form obtained from $(N, v)$ when $j$ delegates her role to $i$. More precisely, when $i$ participates in a given coalition, then $j$ goes along with her. However, when $i$ does not participate in a coalition, we compute the expected value that is obtained when $j$ joins any other coalition updating the beliefs on coalition structures with Bayes' rule. Hence, the delegation agreement between $i$ and $j$ is not symmetric from the point of view of the coalition considered: if it is $S$ - i.e., it is the "active" coalition whose potential worth is being assessed - the agreement is binding; if it is not $S$ i.e., it is another coalition that is only relevant with regard to the externalities it can create on $S$ - the agreement is not binding, and then coalitions are formed according to the probability distributions contained in $\Lambda .{ }^{12}$ This approach covers the situations where contracts between parties are binding contingent upon a certain project being carried out, but are meaningless otherwise. One such example is a coalition agreement between two political parties, which is typically only binding if both parties are in government but has no bite when they are not. Lastly, when no confusion regarding $\Lambda$ arises we denote $\left(N_{-j}, v_{(\Lambda, i j)}\right)$ simply by $\left(N_{-j}, v_{i j}\right)$.

$\Lambda$-DNP Given $\Lambda \in \mathcal{L}$, a value on $\mathcal{G}, \mathrm{f}$, satisfies the $\Lambda$-delegation neutrality property if for every $N \subseteq \Omega,(N, v) \in \mathcal{G}$, and $i, j \in N$, with $i \neq j$,

$$
\mathrm{f}_{i}(N, v)+\mathrm{f}_{j}(N, v)=\mathrm{f}_{i}\left(N_{-j}, v_{(\Lambda, i j)}\right) .
$$

The above property requires that the payoffs allocated by a rule are not affected by agreements of the above type between two players where one player leaves the game delegating her

\footnotetext{
${ }^{11}$ Recall that $\emptyset$ is a member of any partition.

${ }^{12}$ Note that given $S \subseteq N$ and $(S, P) \in E C^{N}$, the reference to $S$ as "active" coalition when we look at $v(S, P)$ actually originates from an interpretation of the coalition structure $P$ per se, with no reference to the game whatsoever. Accordingly, it can be thought that $(S, P)$ represents a situation in which players in $S$ agree to actively participate in $S$, while players in $N \backslash S$ simply organize themselves into $P_{-S}$.
} 
role to another player who remains in the game and represents the two players only when it comes to actively creating a value.

Finally note that in the particular case of two-player games, i.e., when $N=\{i, j\}$ with $i \neq j$, externalities cannot arise as the only partitions are $\{\emptyset,\{i\},\{j\}\}$ and $\{\emptyset,\{i, j\}\}$. Thus the set of games in partition function form coincides in this case with the set of coalitional games. ${ }^{13}$ The property below applies only to two-player games.

2-PSP A value on $\mathcal{G}, \mathrm{f}$, satisfies the 2-player standard payoff property if for every $i, j \in \Omega$ with $i \neq j$ and $(\{i, j\}, v) \in \mathcal{G}^{\{i, j\}}$,

$$
\mathrm{f}_{i}(\{i, j\}, v)=\frac{1}{2}[v(\{i, j\})+v(\{i\})-v(\{j\})] .
$$

A value satisfying 2-PSP gives for two-player games the payoffs prescribed by many common solution concepts like the Shapley value, the Banzhaf value, or the nucleolus.

\subsection{Two characterization results}

In the following we prove a series of results that lead to two theorems in which we characterize each value of the family of $\Lambda$-Banzhaf values by means of $\Lambda$-DNP and either DPP(w) or 2-PSP.

Proposition 3.1. Let $\Lambda \in \mathcal{L}$. Then the $\Lambda$-Banzhaf value satisfies $\Lambda$-DPP, $\Lambda$-DNP, and 2-PSP.

Proof. Let $N \subseteq \Omega,(N, v) \in \mathcal{G}^{N}$, and $i \in N$. First, if $i$ is a $\Lambda$-dummy player in $(N, v)$,

$$
\mathrm{Ba}_{i}^{\Lambda}(N, v)=\frac{1}{2^{n-1}} \sum_{S \subseteq N_{-i}}\left[v^{\Lambda}\left(S_{+i}\right)-v^{\Lambda}(S)\right]=\frac{1}{2^{n-1}} \sum_{S \subseteq N_{-i}} v^{\Lambda}(\{i\})=v^{\Lambda}(\{i\}),
$$

where the first equality holds by definition of $\mathrm{Ba}^{\Lambda}$ and the second equality holds from the fact that $i$ is a $\Lambda$-dummy player in $(N, v)$. Second, let $j \in N_{-i}$. Then for the $\Lambda$-Banzhaf to satisfy $\Lambda$-DNP it is sufficient that

$$
\left(v^{\Lambda}\right)_{i j}=\left(v_{i j}\right)^{\Lambda}
$$

holds, as in this case we have ${ }^{14}$

$$
\begin{aligned}
\operatorname{Ba}_{i}^{\Lambda}(N, v)+\operatorname{Ba}_{j}^{\Lambda}(N, v) & =\operatorname{Ba}_{i}\left(N, v^{\Lambda}\right)+\operatorname{Ba}_{j}\left(N, v^{\Lambda}\right)=\operatorname{Ba}_{i}\left(N_{-j},\left(v^{\Lambda}\right)_{i j}\right) \\
& =\operatorname{Ba}_{i}\left(N_{-j},\left(v_{i j}\right)^{\Lambda}\right)=\operatorname{Ba}_{i}^{\Lambda}\left(N_{-j}, v_{i j}\right),
\end{aligned}
$$

where the first and last equalities hold from the definition of $\mathrm{Ba}^{\Lambda}$, the second equality holds because Ba satisfies the 2-efficiency property for coalitional games as considered in Casajus (2012), and the third equality holds by Eq. (7).

\footnotetext{
${ }^{13}$ The same is trivially true for one-player games.

${ }^{14}$ We denote by $\mathrm{Ba}$ the Banzhaf value of coalitional games. We note that it can be defined for every $(N, v) \in \mathcal{C G}$ by $\operatorname{Ba}(N, v)=\mathrm{Ba}^{\Lambda}(N, v)$, where $\Lambda \in \mathcal{L}$ is arbitrary. The $\{i j\}$-reduced game for coalitional games is then independent of $\Lambda$ and coincides with the one used in Casajus (2012).
} 
Hence it only remains to prove Eq. (7). Let $i, j \in N$, with $i \neq j$, and $S \subseteq N_{-j}$. We distinguish two cases.

Case 1: $i \in S$.

On the one hand,

$$
\left(v^{\Lambda}\right)_{i j}(S)=v^{\Lambda}\left(S_{+j}\right)=\sum_{P^{\prime} \in \mathcal{P}(N): S_{+j} \in P^{\prime}} \lambda^{N}\left(S_{+j}, P^{\prime}\right) \cdot v\left(S_{+j}, P^{\prime}\right) .
$$

On the other hand,

$$
\begin{aligned}
\left(v_{i j}\right)^{\Lambda}(S) & =\sum_{P \in \mathcal{P}\left(N_{-j}\right): S \in P} \lambda^{N_{-j}}(S, P) \cdot v_{i j}(S, P) \\
& =\sum_{P \in \mathcal{P}\left(N_{-j}\right): S \in P} \lambda^{N_{-j}}(S, P) \cdot v\left(S_{+j}, P_{-S} \cup\left\{S_{+j}\right\}\right) .
\end{aligned}
$$

We note that by setting $P^{\prime}=P_{-S} \cup\left\{S_{+j}\right\}$ we define a one-to-one correspondence between the set of partitions $P \in \mathcal{P}\left(N_{-j}\right)$ such that $S \in P$ and the set of partitions $P^{\prime} \in \mathcal{P}(N)$ such that $S_{+j} \in P^{\prime}$. From the two expressions above and since $\Lambda$ satisfies condition a), we deduce that $\left(v^{\Lambda}\right)_{i j}(S)=\left(v_{i j}\right)^{\Lambda}(S)$.

Case 2: $i \notin S$.

On the one hand,

$$
\begin{aligned}
\left(v^{\Lambda}\right)_{i j}(S) & =v^{\Lambda}(S)=\sum_{P \in \mathcal{P}(N): S \in P} \lambda^{N}(S, P) \cdot v(S, P) \\
& =\sum_{P \in \mathcal{P}\left(N_{-j}\right): S \in P}\left(\sum_{T \in P_{-S}} \lambda^{N}\left(S, P_{-T} \cup\left\{T_{+j}\right\}\right) \cdot v\left(S, P_{-T} \cup\left\{T_{+j}\right\}\right)\right),
\end{aligned}
$$

where the third equality follows simply by reorganizing the terms in the sum. On the other hand,

$$
\begin{aligned}
& \left(v_{i j}\right)^{\Lambda}(S)=\sum_{P \in \mathcal{P}\left(N_{-j}\right): S \in P} \lambda^{N_{-j}}(S, P) \cdot v_{i j}(S, P) \\
= & \sum_{P \in \mathcal{P}\left(N_{-j}\right): S \in P} \lambda^{N_{-j}}(S, P) \cdot\left(\frac{\sum_{T \in P_{-S}} \lambda^{N}\left(S, P_{-T} \cup\left\{T_{+j}\right\}\right) \cdot v\left(S, P_{-T} \cup\left\{T_{+j}\right\}\right)}{\sum_{T \in P_{-S}} \lambda^{N}\left(S, P_{-T} \cup\left\{T_{+j}\right\}\right)}\right) \\
= & \sum_{P \in \mathcal{P}\left(N_{-j}\right): S \in P}\left(\frac{\lambda^{N_{-j}}(S, P)}{\sum_{T \in P_{-S}} \lambda^{N}\left(S, P_{-T} \cup\left\{T_{+j}\right\}\right)}\right)\left(\sum_{T \in P_{-S}} \lambda^{N}\left(S, P_{-T} \cup\left\{T_{+j}\right\}\right) \cdot v\left(S, P_{-T} \cup\left\{T_{+j}\right\}\right)\right),
\end{aligned}
$$

where the first (maybe empty) summation after the second equality is taken only for those $P \in \mathcal{P}\left(N_{-j}\right)$ such that $\sum_{T \in P_{-S}} \lambda^{N}\left(S, P_{-T} \cup\left\{T_{+j}\right\}\right)>0 .{ }^{15}$ Since $\Lambda$ satisfies condition b), we obtain $\left(v^{\Lambda}\right)_{i j}(S)=\left(v_{i j}\right)^{\Lambda}(S)$.

Third, and last, it is trivial to check that the $\Lambda$-Banzhaf value satisfies 2-PSP.

\footnotetext{
${ }^{15} \mathrm{By}$ definition the summation over the empty set is zero.
} 
Before we prove the uniqueness part of the characterization results of the $\Lambda$-Banzhaf value it is convenient to show an intermediate lemma.

Lemma 3.1. Let $\Lambda \in \mathcal{L}$. Then, for every $N \subseteq \Omega, i, j \in N$, with $i \neq j$, and $S \subseteq N$, with $S \neq \emptyset,{ }^{16}$

$$
\left(e_{(S, *)}^{N}\right)_{i j}= \begin{cases}e_{(S, *)}^{N_{-j}} & \text { if } i \notin S \text { and } j \notin S, \\ e_{\left(\left(S_{-j}\right)_{+i}, *\right)}^{N_{-j}} & \text { if } i \notin S \text { and } j \in S, \\ e_{\left(S_{-j}, *\right)}^{N_{-j}} & \text { if } i \in S .\end{cases}
$$

Proof. First of all note that $e_{(S, *)}^{N} \in \mathcal{C} \mathcal{G}^{N}$. Indeed, for every $(T, Q) \in E C^{N_{-j}}$,

$$
e_{(S, *)}^{N}(T, Q)=\left\{\begin{array}{lc}
1 & \text { if } S \subseteq T \\
0 & \text { otherwise. }
\end{array}\right.
$$

Hence, we can denote $e_{(S, *)}^{N}(T, Q)$ simply by $e_{(S, *)}^{N}(T)$. We point out that the set $\left\{e_{(S, *)}^{N}: S \subseteq\right.$ $N$ and $S \neq \emptyset\}$ is just the "classical" basis of $\mathcal{C G}$ consisting of unanimity games.

Next, let $T \subseteq N_{-j}$ and distinguish three cases. First, suppose that $i \notin S$ and $j \notin S$. Then,

$$
\left(e_{(S, *)}^{N}\right)_{i j}(T)= \begin{cases}1 & \text { if } S \subseteq T \\ 0 & \text { otherwise. }\end{cases}
$$

Second, suppose that $i \notin S$ and $j \in S$. Then,

$$
\left(e_{(S, *)}^{N}\right)_{i j}(T)=\left\{\begin{array}{ll}
1 & \text { if } S \subseteq T_{+j} \text { and } i \in T, \\
0 & \text { otherwise, }
\end{array}= \begin{cases}1 & \text { if }\left(S_{-j}\right)_{+i} \subseteq T, \\
0 & \text { otherwise. }\end{cases}\right.
$$

Third, suppose that $i \in S$. Then,

$$
\left(e_{(S, *)}^{N}\right)_{i j}(T)=\left\{\begin{array}{cc}
1 & \text { if } S \subseteq T_{+j}, \\
0 & \text { otherwise }
\end{array}=\left\{\begin{array}{lc}
1 & \text { if } S_{-j} \subseteq T, \\
0 & \text { otherwise }
\end{array}\right.\right.
$$

In all three cases, the desired result follows immediately using (9).

Next, for every $\Lambda \in \mathcal{L}$ we provide two characterizations of the $\Lambda$-Banzhaf value in the framework of all games in partition function form.

Proposition 3.2. Let $\Lambda \in \mathcal{L}$. Then, there is at most one value on $\mathcal{G}$ that satisfies $\mathrm{DPP}(\mathrm{w})$ and $\Lambda$-DNP.

Proof. First, we prove uniqueness for two-player games. ${ }^{17}$ W.l.o.g., let $N=\{1,2\} \subseteq \Omega$ and take $(N, v) \in \mathcal{G}^{\{1,2\}}$. Then, there are $\lambda_{1}, \lambda_{2}, \lambda_{12} \in \mathbb{R}$ such that

$$
v=\lambda_{1} \cdot e_{(\{1\}, *)}+\lambda_{2} \cdot e_{(\{2\}, *)}+\lambda_{12} \cdot e_{(N, *)} .
$$

\footnotetext{
${ }^{16}$ For the sake of the notation any reference to $\Lambda$ has been omitted.

${ }^{17}$ This part is based on the proof of Theorem 7 in Casajus (2012).
} 
Let $M=\{1,2,3,4\}$ and $(M, z) \in \mathcal{G}^{M}$ be defined by

$$
z=\left(\lambda_{1}-\lambda_{2}\right) \cdot e_{(\{4\}, *)}+\lambda_{2} \cdot \sum_{l \in\{1,2,3\}}\left(e_{(\{l\}, *)}-e_{(N \backslash\{l, 4\}, *)}\right)+\left(\lambda_{12}+2 \lambda_{2}\right) \cdot e_{(\{1,2,3\}, *)} .
$$

Using (8), it easily follows that

$$
z_{13}=z_{23}=\left(\lambda_{1}-\lambda_{2}\right) \cdot e_{(\{4\}, *)}+\lambda_{2} \cdot\left(e_{(\{1\}, *)}+e_{(\{2\}, *)}\right)+\lambda_{12} \cdot e_{(\{1,2\}, *)} .
$$

Let now $\left(M_{-3}, w\right) \in \mathcal{G}^{M_{-3}}$, where $w=z_{13}$. By $\Lambda$-DNP,

$$
\mathrm{f}_{1}\left(M_{-3}, w\right)=\mathrm{f}_{1}\left(M_{-3}, z_{13}\right)=\mathrm{f}_{1}(M, z)+\mathrm{f}_{3}(M, z)
$$

and

$$
\mathrm{f}_{2}\left(M_{-3}, w\right)=\mathrm{f}_{1}\left(M_{-3}, z_{23}\right)=\mathrm{f}_{2}(M, z)+\mathrm{f}_{3}(M, z) .
$$

From (8) it follows that 1 is a dummy player in the weak sense in $\left(\left(M_{-3}\right)_{-2}, w_{12}\right)$, implying that

$$
\begin{aligned}
2 \lambda_{2}+\lambda_{12} & =\mathrm{f}_{1}\left(\left(M_{-3}\right)_{-2}, w_{12}\right)=\mathrm{f}_{1}\left(M_{-3}, w\right)+\mathrm{f}_{2}\left(M_{-3}, w\right) \\
& =\mathrm{f}_{1}(M, z)+\mathrm{f}_{2}(M, z)+2 \mathrm{f}_{3}(M, z),
\end{aligned}
$$

where the first equality holds by $\operatorname{DPP}(\mathrm{w})$, the second equality by $\Lambda$-DNP, and the last equality is due to Eqs. (11) and (12). As players 1, 2, and 3 play symmetric roles in $z$, we can repeat the procedure above exchanging the roles of the three players in the definition of $z$ to obtain

$$
\mathrm{f}_{1}(M, z)=\mathrm{f}_{2}(M, z)=\mathrm{f}_{3}(M, z)=\frac{\lambda_{2}}{2}+\frac{\lambda_{12}}{4} .
$$

Note that by plugging the above equation into Eqs. (11) and (12), we obtain $\mathrm{f}_{1}\left(M_{-3}, w\right)=$ $\lambda_{2}+\frac{\lambda_{12}}{2}$. As $\left(M_{-3}, w\right) \in \mathcal{C G}^{M_{-3}}$ and 4 is a dummy player in the weak sense in $\left(M_{-3}, w\right)$, by $\operatorname{DPP}(\mathrm{w})$ we have

$$
\mathrm{f}_{4}\left(M_{-3}, w\right)=\lambda_{1}-\lambda_{2} .
$$

Lastly, observe that $\left(M_{-3}\right)_{-4}=\{1,2\}$ and, by Eq. (8),

$$
w_{14}=\lambda_{1} \cdot e_{(\{1\}, *)}+\lambda_{2} \cdot e_{(\{2\}, *)}+\lambda_{12} \cdot e_{(\{1,2\}, *)}=v .
$$

As a consequence,

$$
\mathrm{f}_{1}(N, v)=\mathrm{f}_{1}\left(M_{-3}, w\right)+\mathrm{f}_{4}\left(M_{-3}, w\right)=\lambda_{1}+\frac{\lambda_{12}}{2},
$$

where the first equality holds by $\Lambda$-DNP. Observe that, from (8), we have

$$
v_{12}=\left(\lambda_{1}+\lambda_{2}+\lambda_{12}\right) \cdot e_{(1, *)} .
$$

Since $\left(\{1\}, v_{12}\right) \in \mathcal{G}^{\{1\}}$ and 1 is a dummy player in the weak sense in $\left(\{1\}, v_{12}\right)$,

$$
\lambda_{1}+\lambda_{2}+\lambda_{12}=\mathrm{f}_{1}\left(\{1\}, v_{12}\right)=\mathrm{f}_{1}(N, v)+\mathrm{f}_{2}(N, v),
$$


where the last equality holds by $\Lambda$-DNP. Therefore, $\mathrm{f}_{2}(v)=\lambda_{2}+\frac{\lambda_{12}}{2}$. All in all, we have seen that $\mathrm{f}(N, v)=\mathrm{Ba}(N, v)$.

Second, uniqueness for games $(N, v) \in \mathcal{G}$ with $n=1$ follows trivially from $\Lambda$-DNP, and the fact that the payoffs for two-player games are uniquely determined.

Third, suppose that uniqueness holds for every $\left(N^{\prime}, v\right) \in \mathcal{G}$ where $\left|N^{\prime}\right|<n$ (with $n>2$ ) and let $(N, v) \in \mathcal{G} \cdot{ }^{18}$ By $\Lambda$-DNP, it holds, for every $i, j \in N$,

$$
\mathrm{f}_{i}(N, v)+\mathrm{f}_{j}(N, v)=\mathrm{f}_{i}\left(N_{-j}, v_{i j}\right) .
$$

The right-hand side of Eq. (14) is unique according to the inductive hypothesis. Since Eq. (14) holds for every pair $i, j \in N$, with $i \neq j$, we have a system of $\left(\begin{array}{l}n \\ 2\end{array}\right)$ equations and $n$ unknown variables. It is easy to check that the system has at most one solution. Moreover, by Proposition 3.1 the system is solvable.

A careful analysis of the proof reveals that the result in Proposition 3.2 remains valid even if we restrict the application of $\operatorname{DPP}(\mathrm{W})$ only to games $(N, v) \in \mathcal{G}$ where $n \leq 4$. The following theorem is a straightforward consequence of the previous results.

Theorem 3.1. Let $\Lambda \in \mathcal{L}$. Then the $\Lambda$-Banzhaf value is the only value on $\mathcal{G}$ that satisfies $\Lambda$-DNP and $\operatorname{DPP}(\mathrm{W}) .{ }^{19}$

Proof. Trivial from Propositions 3.1 and 3.2, and the fact that $\Lambda$-DPP implies DPP(w).

The next result shows that the $\Lambda$-Banzhaf value can be characterized without the need for the dummy player property.

Theorem 3.2. Let $\Lambda \in \mathcal{L}$. Then the $\Lambda$-Banzhaf value is the only value on $\mathcal{G}$ that satisfies $\Lambda$-DNP and 2-PSP. ${ }^{20}$

Proof. As in the proof of Proposition 3.2, uniqueness can be shown by induction. The case $n=2$ follows immediately from 2-PSP. The case $n=1$ then follows from the case $n=2$ by $\Lambda$-DNP. The inductive reasoning for $n \geq 2$ is the same as in the proof of Proposition 3.2, as it only depends on $\Lambda$-DNP.

\section{An Ordinal Approach and Simple Games}

In Section 2 we have seen that to calculate the $\Lambda$-Banzhaf value of a game $(N, v)$, all the information contained in $\lambda^{N}$ is used (through the definition of $\left(N, v^{\Lambda}\right)$ ). Consequently, changes in $\lambda^{N}$ that preserve the relative order of coalitions regarding the probability that they occur

\footnotetext{
${ }^{18}$ This part is based on Lehrer (1988).

${ }^{19}$ The independence of the axioms can be proved analogously as in Casajus (2012).

${ }^{20}$ The independence of the axioms is trivial.
} 
may lead to very different payoffs for the players as allocated by the $\Lambda$-Banzhaf value. Such a feature might be undesirable if probabilities cannot be reliably estimated. In this section, we assume that only the relative order of the coalition structures regarding their corresponding probability is meaningful. More specifically, we propose a second family of values, one for each $\Lambda$, that are immune to changes in $\lambda^{N}$ that do not alter which the most likely coalition structure is for any given set. Accordingly, we name an arbitrary element in the new family as the ordinal $\Lambda$-Banzhaf value. In the absence of externalities the two families of values considered in the paper collapse to the Banzhaf value for coalitional games.

The approach in this section yields an additional advantage. In the case of the ordinal $\Lambda$-Banzhaf value (unlike that of the $\Lambda$-Banzhaf value) we are able to provide characterization results not only within the framework of all partition function form games (as in the case of the $\Lambda$-Banzhaf value) but also within the framework of a particular, relevant subclass of games in partition function form, the so-called simple games in partition function form. The class of simple games in partition function form comprises games in which the worth of any embedded coalition takes only one of two values. To be consistent with the literature on simple coalitional games we also require a certain notion of monotonicity. Formally, for each $(N, v) \in \mathcal{G}$, we consider the following properties:

(i) For every $(S, P) \in E C^{N}, v(S, P) \in\{0,1\}$,

(ii) $v(N,\{\emptyset, N\})=1$,

(iii) For every $S, T \subseteq N$, with $S \subseteq T$, and $(S, P) \in E C^{N}, v(S, P) \leq v(T,(P \backslash T) \cup\{T\})$.

First, (i) requires that an embedded coalition is either winning (and the value is 1) or losing (and the value is 0 ). Second, (ii) requires that the grand coalition is always winning. Third, (iii) requires that a winning embedded coalition $S$ can never become losing when more players join it, with the remaining players organized as before. A game $(N, v) \in \mathcal{G}$ is a simple game in partition function form (just a simple game) if it satisfies (i), (ii), and (iii). We let $\mathcal{S G}$ be the subset of $\mathcal{G}$ that consists of simple games. Note that $\mathcal{S G}$ contains all simple coalitional games. ${ }^{21}$

We point out that the class of simple games considered here allows for two coalitions of a given partition to be simultaneously winning. While this feature might be a bit counterintuitive, it is a necessary condition for a class of $\{0,1\}$-valued games to encompass the parliamentary situations described in the Introduction, where relative majorities instead of fixed majorities

\footnotetext{
${ }^{21}$ All the results contained in this section remain valid if we dispense with condition (iii) and hence do not impose any monotonicity condition in the definition of $\mathcal{S G}$.
} 
may be considered. ${ }^{22}$

In the remaining part of this section we do three things. First, we present a collusion neutrality property in the spirit of $\Lambda$-DNP that only takes into account ordinal information included in $\Lambda$. This property - which we call the ordinal $\Lambda$-delegation neutrality property - is meaningful within the framework of simple games, provided that $\Lambda$ satisfies some mild condition. This is in sharp contrast with what occurs with $\Lambda$-DNP: Indeed, $\Lambda$-DNP fails to be equally meaningful since it makes use of a collusion transformation for which the set of simple games is not closed. Second, for each $\Lambda$ we define a new value for arbitrary partition function form games which we call the ordinal $\Lambda$-Banzhaf value. These values only take into account ordinal information included in $\Lambda$. Third, we show that the ordinal $\Lambda$-Banzhaf value is the only value - either within the framework of simple games or that of all games - that satisfies the ordinal $\Lambda$-delegation neutrality property and either DPP(W) or 2-PSP.

\subsection{A different amalgamation neutrality property}

To define the new amalgamation neutrality property it is convenient to assume henceforth two technical requirements on the probability distributions given by $\Lambda=\left\{\lambda^{N}: N \subseteq \Omega\right\}$.

c) Given $N \subseteq \Omega$ and $S \subseteq N$, there exists a unique $P^{N, S} \in \mathcal{P}(N)$ with $S \in P^{N, S}$ such that

$$
\left\{P^{N, S}\right\}=\underset{P \in \mathcal{P}(N): S \in P}{\arg \max } \lambda^{N}(S, P) .
$$

d) Given $N \subseteq \Omega, j \in N$, and $(S, P) \in E C^{N_{-j}}$ such that

$$
P \in \underset{P^{\prime} \in \mathcal{P}\left(N_{-j}\right): S \in P^{\prime}}{\arg \max } \lambda^{N_{-j}}\left(S, P^{\prime}\right),
$$

there exists a unique $T^{N, j,(S, P)} \in P_{-S}$ such that

$$
\left\{T^{N, j,(S, P)}\right\}=\underset{T \in P_{-S}}{\arg \max } \lambda^{N}\left(S, P_{-T} \cup\left\{T_{+j}\right\}\right) .
$$

First, c) requires that for each set there is always a unique coalition structure which is the most likely configuration among all possible coalition structures. Second, d) requires that given

\footnotetext{
${ }^{22}$ For instance, consider the following example. There are three parties in a parliament, $N=\{1,2,3\}$, and the seats are distributed as follows: Parties 1 and 2 have both 20 seats and Party 3 has 15 seats. Obviously, no party has an absolute majority. If absolute majority is required, then the three parties are in fairly symmetric position. This is indeed captured by the simple coalitional game $(N, v)$ where $v(\{k\})=0$ and $v\left(N_{-k}\right)=v(N)=1$ for all $k \in N$. However, if only a relative majority is needed and we consider $\Lambda^{* *}$ - i.e. no parties make any agreement when they are in the opposition - then clearly Parties 1 and 2 are in a better position than Party 3. The only way to capture this by means of a game where the values of embedded coalitions are either 0 or 1 , is by letting $v(\{1\}, * *)=v(\{2\}, * *)=1$ and $v(\{3\}, * *)=0$. That is, $\mathcal{S G}$ must necessarily contain games where there are two or more winning coalitions in the same partition.
} 
$S \subseteq N_{-j}$, when player $j$ incorporates into a most likely coalition structure $P_{-S} \in \mathcal{P}\left(N_{-j} \backslash S\right)$ that may arise for the set $N_{-j} \backslash S$, there is exactly one coalition $T \in P_{-S}$ that has the highest ex-ante probability among all other coalitions in $P_{-S}$ that player $j$ will join it. Conditions c) and $\mathbf{d}$ ) are thus meaningful if we assume the principle that only the most likely configurations matter, as both conditions limit to a singleton the set of most likely coalition structures for two different scenarios respectively: condition c) in the case where all coalition structures of a set are taken into account; condition $\mathbf{d}$ ) in the case where a partition is already given and there is an outside player that joins one of the existing coalitions. Note that no restriction is imposed on those configurations that are not the most likely. Additionally, we introduce - and assume henceforth - two notions for consistency in the beliefs that take into account only ordinal considerations.

e) $\Lambda$ is ordinally consistent, i.e., for every $N \subseteq \Omega, j \in N$, and $S \subseteq N_{-j}$,

$$
\underset{P \in \mathcal{P}(N): S \in P}{\arg \max } \lambda^{N}(S, P) \subseteq\left\{P_{-T}^{\prime} \cup\left\{T_{+j}\right\}: T \in P_{-S}^{\prime}, P^{\prime} \in \underset{P^{\prime \prime} \in \mathcal{P}\left(N_{-j}\right): S \in P^{\prime \prime}}{\arg \max } \lambda^{N_{-j}}\left(S, P^{\prime \prime}\right)\right\} .
$$

f) Given $N \subseteq \Omega, j \in N, S, T \subseteq N_{-j}$ with $S \subseteq T$, and $(S, P) \in E C^{N_{-j}}$,

$$
\begin{aligned}
& \underset{R^{\prime \prime} \in P \backslash T}{\arg \max } \lambda^{N}\left(T,((P \backslash T) \cup\{T\})_{-R^{\prime \prime}} \cup\left\{R_{+j}^{\prime \prime}\right\}\right) \\
& \subseteq\left\{R^{\prime} \backslash T: R^{\prime} \in \underset{R \in P_{-S}}{\arg \max } \lambda^{N}\left(S, P_{-R} \cup\left\{R_{+j}\right\}\right)\right\} .
\end{aligned}
$$

On the one hand, if we assume conditions $\mathbf{c}$ ) and $\mathbf{d}$ ) then $\Lambda$ is ordinally consistent for a given $S \subseteq N_{-j}$ if the most likely configuration of players in $N_{-j} \backslash S$ according to $\Lambda$ is independent of whether or not we consider player $j$, with $j \in N .{ }^{23}$ Indeed, condition e) requires that the most likely coalition structure that contains $S$ as a coalition has to be of the following type: first, the most likely partition of $N_{-j}$ that contains $S$ as a coalition is singled out; second, among all the coalitions of the latter partition different than $S$, player $j$ is added to the coalition with the ex ante highest probability that she joins it. On the other hand, condition f) relates the set of coalitions which are ex ante more likely that $j$ joins in two different situations: one where all coalitions in $P_{-S} \backslash T$, or equivalently $P \backslash T$, are available (left-hand side of the equation in f)); one where all coalitions in $P_{-S}$ are available (right-hand side of the equation in $\mathbf{f}$ )). More specifically, it requires the following: every element in the set of the first situation has to be obtained from an element in the set of the second situation by removing from the latter the agents that belong to $T$. In other words, condition f) requires that the set of ex ante most likely coalitions for $j$ to join can never become larger as some agents are ruled out from consideration.

\footnotetext{
${ }^{23}$ Without conditions $\mathbf{c}$ ) and $\mathbf{d}$ ) the interpretation is similar but uniqueness of the most likely configurations is not guaranteed.
} 
Lastly, we let $\tilde{\mathcal{L}}$ denote the set of $\Lambda$ that satisfy conditions a), c), d), e), and $\mathbf{f}$ ). We stress that $\tilde{\mathcal{L}} \neq \emptyset$ as it contains the $\Lambda$ defined in Examples 2.1, provided that $p \neq \frac{1}{2}$, and 2.2.

We are now in a position to introduce the game, which we name ordinal $\{i j\}$-reduced game and is denoted by $\left(N_{-j}, \tilde{v}_{(\Lambda, i j)}\right) \in \mathcal{G}^{N_{-j}}$ and defined, for every $(S, P) \in E C^{N_{-j}}$, by

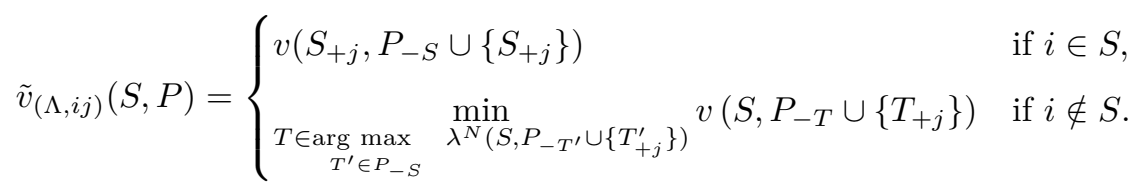

As in the reduced game defined in Eq. (5), the delegation agreement between $i$ and $j$ is only binding when they join the "active" coalition $S$. When that is not the case, we assume that among those coalitions of $P_{-S}$ that are most likely that $j$ incorporates to, player $j$ joins a coalition in a way that the value created by $S$ is minimum. ${ }^{24}$ Also note that different $\Lambda$ define the same amalgamation game when the relative likelihood that $j$ joins the coalitions in $P$ remains unaltered, hence justifying the name of the reduced game. When no confusion arises, we write $\left(N_{-j}, \tilde{v}_{i j}\right)$ instead of $\left(N_{-j}, \tilde{v}_{(\Lambda, i j)}\right)$. Next, we show that the ordinal $\{i j\}$-reduced game is simple when the original game is simple.

Lemma 4.1. Let $\Lambda \in \tilde{\mathcal{L}}$. Then, for every $N \subseteq \Omega,(N, v) \in \mathcal{S G}$, and $i, j \in N$, with $i \neq j$, we have $\left(N_{-j}, \tilde{v}_{(\Lambda, i j)}\right) \in \mathcal{S G}$.

Proof. On the one hand, $\left(N_{-j}, \tilde{v}_{i j}\right)$ trivially satisfies (i) and (ii). On the other hand, to prove (iii) we distinguish three cases. Let $S, T \subseteq N_{-j}$, with $S \subseteq T$, and $(S, P) \in E C^{N_{-j}}$. First, if $i \in S$,

$$
\begin{aligned}
\tilde{v}_{i j}(S, P) & =v\left(S_{+j}, P_{-S} \cup\left\{S_{+j}\right\}\right) \leq v\left(T_{+j},\left(P \backslash T_{+j}\right) \cup\left\{T_{+j}\right\}\right) \\
& =v\left(T_{+j},(P \backslash T) \cup\left\{T_{+j}\right\}\right)=\tilde{v}_{i j}(T,(P \backslash T) \cup\{T\}),
\end{aligned}
$$

where the inequality holds since $(N, v)$ satisfies (iii), the penultimate equality holds since $P \in$ $\mathcal{P}\left(N_{-j}\right)$, and the last equality follows from $i \in T$. Second, if $i \notin S$ and $i \in T$,

$$
\begin{aligned}
& \tilde{v}_{i j}(S, P)=\min _{R \in\left\{\underset{R^{\prime} \in P_{-S}}{\arg \max } \lambda^{N}\left(S, P_{-R^{\prime}} \cup\left\{R_{+j}^{\prime}\right\}\right)\right\}}^{v\left(S, P_{-R} \cup\left\{R_{+j}\right\}\right)} \\
& \left.\leq \quad \min _{R \in\left\{\underset{R^{\prime} \in P_{-S}}{\arg \max }\right.}^{\lambda^{N}\left(S, P_{-R^{\prime}} \cup\left\{R_{+j}^{\prime}\right\}\right)}\right\} v\left(T_{+j},\left(\left(P_{-R} \cup\left\{R_{+j}\right\}\right) \backslash T_{+j}\right) \cup\left\{T_{+j}\right\}\right) \\
& =v\left(T_{+j},(P \backslash T) \cup\left\{T_{+j}\right\}\right)=\tilde{v}_{i j}(T,(P \backslash T) \cup\{T\}),
\end{aligned}
$$

\footnotetext{
${ }^{24}$ Taking the minimum is relevant only when the set $\left\{\underset{T^{\prime} \in P_{-S}}{\arg \max } \lambda^{N}\left(S, P_{-T^{\prime}} \cup\left\{T_{+j}^{\prime}\right\}\right)\right\}$ is not a singleton.
} This occurs, for instance, in the $\Lambda$ considered in Examples 2.1 and 2.2. 
where the inequality holds since $(N, v)$ satisfies (iii) and the second equality holds since the left-hand side is independent of $R$. Third, if $i \notin T$,

$$
\begin{aligned}
& \left.\tilde{v}_{i j}(S, P)=\min _{R \in\left\{\underset{R^{\prime} \in P_{-S}}{\arg \max }\right.}^{\lambda^{N}\left(S, P_{-R^{\prime}} \cup\left\{R_{+j}^{\prime}\right\}\right)}\right\} v\left(S, P_{-R} \cup\left\{R_{+j}\right\}\right) \\
& \leq \min _{R \in\left\{\underset{R^{\prime} \in P_{-S}}{\arg \max } \lambda^{N}\left(S, P_{-R^{\prime}} \cup\left\{R_{+j}^{\prime}\right\}\right)\right\}} v\left(T,\left(\left(P_{-R} \cup\left\{R_{+j}\right\}\right) \backslash T\right) \cup\{T\}\right) \\
& \leq \underset{R \in\left\{\underset{R^{\prime \prime} \in P \backslash T}{\arg \max } \lambda^{N}\left(T,((P \backslash T) \cup\{T\})_{-R^{\prime \prime}} \cup\left\{R_{+j}^{\prime \prime}\right\}\right)\right\}}{\min } v\left(T,((P \backslash T) \cup\{T\})_{-R} \cup\left\{R_{+j}\right\}\right) \\
& =\tilde{v}_{i j}(T,\{P \backslash T\} \cup\{T\}),
\end{aligned}
$$

where the first equality follows from $i \notin S$ and the first inequality holds since $(N, v)$ satisfies (iii). Moreover, the second inequality holds by condition $\mathbf{f}$ ) and the definition of the ordinal $\{i j\}$-reduced game - see Eq. (15). Indeed, note that given $R^{\prime} \in P_{-S}$ and $R^{\prime \prime} \in P \backslash T$ such that $R^{\prime \prime}=R^{\prime} \backslash T$,

$$
\left(\left(P_{-R^{\prime}} \cup\left\{R_{+j}^{\prime}\right\}\right) \backslash T\right) \cup\{T\}=((P \backslash T) \cup\{T\})_{-R^{\prime \prime}} \cup\left\{R_{+j}^{\prime \prime}\right\} .
$$

Next, we consider the following amalgamation neutrality property.

$\Lambda$-DNP(O) Given $\Lambda \in \tilde{\mathcal{L}}$, a value on $\mathcal{G}, \mathrm{f}$, satisfies the ordinal $\Lambda$-delegation neutrality property if for every $N \subseteq \Omega,(N, v) \in \mathcal{G}$, and $i, j \in N$, with $i \neq j$,

$$
\mathrm{f}_{i}(N, v)+\mathrm{f}_{j}(N, v)=\mathrm{f}_{i}\left(N_{-j}, \tilde{v}_{(\Lambda, i j)}\right) .
$$

The interpretation of $\Lambda$-DNP(O) is analogous to the case of $\Lambda$-DNP, the only difference being that the reduced game takes into account now only the ordinal information contained in $\Lambda$.

\subsection{Another family of values}

In the following, we introduce a new family of values for arbitrary games, although we initially focus our attention on simple games. To do so, given $(N, v) \in \mathcal{G}$ and $\Lambda \in \tilde{\mathcal{L}}$ we introduce the game $\left(N, \tilde{v}^{\Lambda}\right)$ defined for every $S \subseteq N$ by

$$
\tilde{v}^{\Lambda}(S)=v\left(S, P^{N, S}\right)
$$

where $P^{N, S}$ is introduced in condition $\left.\mathbf{c}\right)$. Note that different $\Lambda$ define the same coalitional game $\left(N, \tilde{v}^{\Lambda}\right)$ as long as modifications in $\lambda^{N}$ do not change $P^{N, S}$. We can now define a new family of values, one for every $\Lambda \in \tilde{\mathcal{L}}$. 
Definition 4.1. Given $\Lambda \in \tilde{\mathcal{L}}$, the ordinal $\Lambda$-Banzhaf value, $\widetilde{\mathrm{Ba}^{\Lambda}}$, is the value defined for every $(N, v) \in \mathcal{G}$ and $i \in N$ by

$$
\widetilde{\mathrm{Ba}}_{i}^{\Lambda}(N, v)=\frac{1}{2^{n-1}} \sum_{S \subseteq N_{-i}}\left[\tilde{v}^{\Lambda}\left(S_{+i}\right)-\tilde{v}^{\Lambda}(S)\right] .
$$

It is immediate to check that when there are no externalities, i.e., $(N, v) \in \mathcal{S G}$, we have $\widetilde{\mathrm{Ba}^{\Lambda}}(N, v)=\mathrm{Ba}^{\Lambda}(N, v)$. For arbitrary games, however, both values are in general different.

\subsection{More characterization results}

We are now in a position to present two characterizations of every ordinal $\Lambda$-Banzhaf value on the class of simple games.

Theorem 4.1. Let $\Lambda \in \tilde{\mathcal{L}}$. Then the ordinal $\Lambda$-Banzhaf value is the only value on $\mathcal{S G}$ that satisfies $\Lambda$-DNP(O) and $\mathrm{DPP}(\mathrm{W})$.

Proof. First, to prove the existence we can simply repeat the proof of Proposition 3.1, which now hinges on

$$
{\widetilde{\left(\tilde{v}^{\Lambda}\right)_{i j}}}={\widetilde{\left(\tilde{v}_{i j}\right)}}^{\Lambda}
$$

In order to prove Eq. (17), let $i, j \in N$, with $i \neq j$, and $S \subseteq N_{-j}$. We distinguish two cases.

Case 1: $i \in S$.

In this case, let $P^{*}:=P^{N_{-j}, S} \in \mathcal{P}\left(N_{-j}\right)$ as defined in condition c). Then $S \in P^{*}$ and

$$
\begin{aligned}
\left\{P_{-S}^{*}\right\} & =\underset{P_{-S} \in \mathcal{P}\left(N_{-j} \backslash S\right)}{\arg \max } \lambda^{N_{-j}}\left(S, P_{-S} \cup\{S\}\right) \\
& =\underset{P_{-S} \in \mathcal{P}\left(N \backslash S_{+j}\right)}{\arg \max } \lambda^{N}\left(S_{+j}, P_{-S} \cup\left\{S_{+j}\right\}\right)=\left\{P_{-S}^{*}\right\},
\end{aligned}
$$

where the second equality holds by condition a). On the one hand, from the third equality in Eq. (18) we obtain

$$
\widetilde{\left(\tilde{v}^{\Lambda}\right)_{i j}}(S)=\tilde{v}^{\Lambda}\left(S_{+j}\right)=v\left(S_{+j}, P_{-S}^{*} \cup\left\{S_{+j}\right\}\right)
$$

On the other hand, from the first equality in Eq. (18) we obtain

$$
{\widetilde{\left(\tilde{v}_{i j}\right.}}^{\Lambda}(S)=\tilde{v}_{i j}\left(S, P^{*}\right)=v\left(S_{+j}, P_{-S}^{*} \cup\left\{S_{+j}\right\}\right) .
$$

Therefore, Eq. (17) holds.

Case 2: $i \notin S$.

As in the previous case, let $P^{*}:=P^{N_{-j}, S} \in \mathcal{P}\left(N_{-j}\right)$ as defined in condition c). I.e., $S \in P^{*}$ and

$$
\left\{P^{*}\right\}=\underset{P \in \mathcal{P}\left(N_{-j}\right): S \in P}{\arg \max } \lambda^{N_{-j}}(S, P) .
$$


Additionally, let $P^{* *}:=P^{N, S} \in \mathcal{P}(N)$ as defined also in condition c). I.e., $S \in P^{* *}$ and

$$
\left\{P^{* *}\right\}=\underset{P \in \mathcal{P}(N): S \in P}{\arg \max } \lambda^{N}(S, P) .
$$

On the one hand, from Eq. (19) it follows that

$$
{\widetilde{\left(\tilde{v}^{\Lambda}\right)_{i j}}}_{(S)}=\tilde{v}^{\Lambda}(S)=v\left(S, P^{* *}\right) .
$$

On the other hand, from Eq. (20) it follows that

$$
{\widetilde{\left(\tilde{v}_{i j}\right)}}^{\Lambda}(S)=\tilde{v}_{i j}\left(S, P^{*}\right)=v\left(S, P_{-T^{*}}^{*} \cup\left\{T_{+j}^{*}\right\}\right),
$$

where $T^{*}=T^{N, j,\left(S, P^{*}\right)}$ as defined in condition $\left.\mathbf{d}\right)$, i.e.,

$$
\left\{T^{*}\right\}=\underset{T \in P_{-S}^{*}}{\arg \max } \lambda^{N}\left(S, P_{-T}^{*} \cup\left\{T_{+j}\right\}\right) .
$$

Lastly, from condition e) it follows that $P^{* *}=P_{-T^{*}}^{*} \cup\left\{T_{+j}^{*}\right\}$. As a consequence, Eq. (17) holds.

Second, by repeating the induction argument in the proof of Theorem 3.1, to prove uniqueness it suffices to focus our attention on simple games $(N, v) \in \mathcal{S G}$ with $n \leq 2$. On the one hand, the case where $n=1$ follows immediately from $\operatorname{DPP}(\mathrm{w})$. On the other hand, when $n=2$ there exist only four different games which are simple. Indeed, let w.l.o.g. $N=\{1,2\}$ and consider a value $\mathrm{f}$ on $\mathcal{S G}$ that satisfies $\Lambda$-DNP $(\mathrm{O})$ and $\operatorname{DPP}(\mathrm{w})$. Let also $\left(N, u_{\emptyset}\right) \in \mathcal{S G}$ be defined, for every $(S, P) \in E C^{N}$, by

$$
u_{\emptyset}(S, P)=\left\{\begin{array}{lc}
1 & \text { if } S \neq \emptyset, \\
0 & \text { otherwise. }
\end{array}\right.
$$

Straightforwardly we have that $\left(N, u_{\emptyset}\right),\left(N, e_{(\{1\}, *)}\right),\left(N, e_{(\{2\}, *)}\right)$, and $\left(N, e_{(N, *)}\right)$ are the only simple games with player set $N$. Since $n=2$, they are all coalitional games. Moreover, for coalitional games, $\Lambda$-DNP(O) implies symmetry for any $\Lambda .{ }^{25}$ Since $\left(N_{-j}, \widetilde{v}_{i j}\right)$, with $\{i, j\}=N$, is a simple game defined on a singleton player set, from the fact that $f$ satisfies $\Lambda$-DNP(O) and $\operatorname{DPP}(\mathrm{w})$ it immediately follows that $f$ must coincide with the ordinal $\Lambda$-Banzhaf value for the four games considered.

Following the main lines of the proof of the above theorem - with small changes due to the use of 2-PSP instead of $\operatorname{DPP}(\mathrm{W})$ similar to those in the proof of Theorem $3.2-$, we also obtain the next result.

Theorem 4.2. Let $\Lambda \in \tilde{\mathcal{L}}$. Then the ordinal $\Lambda$-Banzhaf value is the only value on $\mathcal{S G}$ that satisfies $\Lambda$-DNP(O) and 2-PSP. ${ }^{26}$

\footnotetext{
${ }^{25}$ See Theorem 1 in Casajus (2012). A value on $\mathcal{S G}, \mathrm{f}$, is symmetric if for every $(N, v) \in \mathcal{S G}, v\left(S_{+i}\right)=v\left(S_{+j}\right)$ for all $S \subseteq N \backslash\{i, j\}$ implies $\mathrm{f}_{i}(N, v)=\mathrm{f}_{j}(N, v)$.

${ }^{26}$ The independence of the axioms is trivial.
} 
Since the probability distributions given by $\Lambda \in \tilde{\mathcal{L}}$ are independent of the game, the ordinal Banzhaf value is additive, i.e., given $N \subseteq \Omega$ we have $\widetilde{\operatorname{Ba}}^{\Lambda}(N, v+w)=\widetilde{\operatorname{Ba}}^{\Lambda}(N, v)+\widetilde{\operatorname{Ba}}^{\Lambda}(N, w)$ for any $(N, v),(N, w) \in \mathcal{G}^{N}$, where $(v+w)(S, P)=v(S, P)+w(S, P)$ for all $(S, P) \in E C^{N}$. Moreover, due to the fact that the games defined in (1) constitute a basis of the vector space of all partition function games with common player set $N$ and, additionally, they are simple games, the characterization results in Theorems 4.1 and 4.2 can be extended to the framework of all games in partition function form.

Corollary 4.1. Let $\Lambda \in \tilde{\mathcal{L}}$. Then the ordinal $\Lambda$-Banzhaf value is the only value on $\mathcal{G}$ that satisfies $\Lambda$-DNP(O) and $\mathrm{DPP}(\mathrm{W})$.

Corollary 4.2. Let $\Lambda \in \tilde{\mathcal{L}}$. Then the ordinal $\Lambda$-Banzhaf value is the only value on $\mathcal{G}$ that satisfies $\Lambda$-DNP(O) and 2-PSP.

In combination with Theorems 3.1 and 3.2 , the results in this section permit us to compare the two families of values introduced in this paper. ${ }^{27}$ On the one hand, we see that demanding $\Lambda$-DNP(O) leads to a value, namely $\widetilde{\mathrm{Ba}^{\Lambda}}$, defined in a way that the information contained in $\Lambda$ is aggregated too abruptly, as opposed to $\mathrm{Ba}^{\Lambda}$, where the information contained in $\Lambda$ is aggregated following a smoother approach. Additionally, when restricted to simple games $\mathrm{Ba}^{\Lambda}$ assigns to any player the probability conditional on $\Lambda$ that she is pivotal in changing the outcome of the game. On the other hand, $\widetilde{\mathrm{Ba}}^{\Lambda}(N, v)$, but not $\mathrm{Ba}^{\Lambda}(N, v)$, is under mild assumptions unresponsive to modifications in $\Lambda$ - e.g. due to measurement mistakes - that do not change which the most likely coalition structure is for any set of players. Therefore, the ordinal $\Lambda$-Banzhaf value is especially relevant when only the ordinal information contained in $\Lambda$ is meaningful. Furthermore, the two characterizations of $\widetilde{\mathrm{Ba}}^{\Lambda}$ presented above are valid for the class of all games as well as for the class of simple games. All in all, depending on the specific setting in which we want to consider our values, either one or other of the families of values could be more suitable.

Lastly, Theorem 4.2 is particularly interesting from the point of view of the study of the distribution of power in legislatures. Indeed, we say that $i \in N$ has veto power within $(N, v)$ if $v(\{S, P\})=1$ implies $i \in S$. Then consider the following property that a value on $\mathcal{S G}$ might satisfy:

2-VPS A value on $\mathcal{S G}, \mathrm{f}$, satisfies the veto property for unilateral and bilateral systems if for every $N \subseteq \Omega$ with $n \leq 2$ and $(N, v) \in \mathcal{S G}$ where exactly one player, $i \in N$, has veto power,

$$
\mathrm{f}_{i}(N, v)=v(N,\{\emptyset, N\}) .
$$

The appeal of the above property within two-party systems is quite obvious - see e.g. May (1952). Corollary 4.3 below reveals that if we require the latter property to hold for one- and

\footnotetext{
${ }^{27}$ We stress that $\mathcal{L} \cap \tilde{\mathcal{L}} \neq \emptyset$ as both sets contain the $\Lambda$ defined in Examples 2.1 - except if $p=\frac{1}{2}-$ and 2.2 , so a proper comparison between values belonging to the two families can be established. Note that while $\mathrm{Ba}^{\Lambda}$ and $\widetilde{\mathrm{Ba}^{\Lambda}}$ coincide for $\Lambda \in\left\{\Lambda^{*}, \Lambda^{* *}\right\}$, both values differ in general for $\Lambda^{p}$.
} 
two-party systems and we want the distribution of power to be (on expectation according to $\Lambda$ ) unaffected by the party agreements that may be subscribed to within a multi-party system, only one possibility remains: the ordinal $\Lambda$-Banzhaf value.

Corollary 4.3. Let $\Lambda \in \tilde{\mathcal{L}}$. Then the ordinal $\Lambda$-Banzhaf value is the only value on $\mathcal{S G}$ that satisfies $\Lambda$-DNP(O) and 2 -VPs. ${ }^{28}$

Proof. By following the main lines of the induction argument in the proof of Theorem 4.1, we can focus our attention on simple games $(N, v) \in \mathcal{S G}$ with $n=2$, as uniqueness when $n=1$ follows immediately from 2-VPS. Recall that when $n=2$ there are only four simple games - see the proof of Theorem 4.1. Also recall that since $n=2$, they are all coalitional games, and that for coalitional games, $\Lambda$-DNP(O) implies symmetry for any $\Lambda$. In particular, for every $N$ with $n=2$ and $(N, v) \in \mathcal{S G}$ where either all players have veto power or no player does, $\mathrm{f}_{i}(N, v)=\mathrm{f}_{j}(N, v)$ for all $i, j \in N$. That is, a value that satisfies $\Lambda$-DNP(O) and 2-VPs within $\mathcal{S G}$ is uniquely determined for the four games considered.

\section{Conclusion}

To date, the number of studies on the Banzhaf value in the presence of externalities has been extremely limited. The present paper provides some new insights from this perspective by introducing two families of values for partition function form games. The proposed values are based on collections of probability distributions over the set of coalition structures. These probability distributions can be understood as beliefs or estimates as to which coalition structures may arise. The first family of values is constructed so that each value takes into account all the information contained in the corresponding collection of probability distributions. In this way it is possible to assess how much each coalition can be expected to be worth on average. Such an approach can be defined as cardinal. By contrast, the second family of values is constructed so that each value considers only the most likely coalition configuration, disregarding the rest of the information contained in the probability distribution. Such an approach can be defined as ordinal.

In this paper, every value of the two families has been characterized by means of two properties. The common feature of all the characterizations has been the use of an amalgamation neutrality property. Such properties build on the definition of a reduced game that emerges once a player leaves the game by delegating her role to another player who stays in the game. We consider the agreement between the two players to be binding only when it comes to create worth, and we assume that they may act independently otherwise.

The solutions proposed and the results presented in this paper have particular value in explaining the distribution of power in legislatures as outlined in the Introduction. Future

\footnotetext{
${ }^{28}$ The independence of the axioms is trivial.
} 
research could be usfully directed at studying further properties of the values introduced here, considering other possible generalizations of the Banzhaf value, as well as identifying empirical applications.

\section{Acknowledgements}

We would like to thank two referees for their comments and suggestions which helped to substantially improve a previous version of the document. All remaining errors are our own responsibility. This research received financial support from the Spanish Ministry of Economy and Competitiveness through Projects ECO2011-22765 and MTM2011-27731-02.

\section{References}

Albizuri, M., Arin, J., and Rubio, J. (2005). An axiom system for games in partition function form. International Game Theory Review, 7:63-72.

Alonso-Meijide, J. M., Álvarez-Mozos, M., and Fiestras-Janeiro, M. G. (2012). Notes on a comment on 2-efficiency and the Banzhaf value. Applied Mathematics Letters, 25(7):10981100 .

Banzhaf, J. F. (1964). Weighted voting doesn't work: A mathematical analysis. Rutgers Law Review, 19:317.

Bolger, E. (1983). The Banzhaf index for multicandidate presidential elections. SIAM Journal of Algebraic Discrete Methods, 4:422-458.

Bolger, E. (1986). Power indices for multicandidate voting games. International Journal of Game Theory, 15:175-186.

Bolger, E. (1990). A characterization of an extension of the Banzhaf value for multicandidate voting games. SIAM Journal of Discrete Mathematics, 3:466-477.

Bolger, E. (2002). Characterizations of two power indices for voting games with $\mathrm{r}$ alternatives. Social Choice and Welfare, 19(4):709-721.

Casajus, A. (2012). Amalgamating players, symmetry, and the Banzhaf value. International Journal of Game Theory, 41:497-515.

de Clippel, G. and Serrano, R. (2008). Marginal contributions and externalities in the value. Econometrica, 76(6):1413-1436.

Dutta, B., Ehlers, L., and Kar, A. (2010). Externalities, potential, value and consistency. Journal of Economic Theory, 145:23802411. 
Freixas, J. and Zwicker, W. (2003). Weighted voting, abstention, and multiple levels of approval. Social Choice and Welfare, 21:399-431.

Hafalir, I. (2007). Efficiency in coalition games with externalities. Games and Economic Behavior, 61:242-258.

Haller, H. (1994). Collusion properties of values. International Journal of Game Theory, 23:261281.

Hart, S. and Mas-Colell, A. (1989). Potential, value, and consistency. Econometrica, 57:589-614.

Lehrer, E. (1988). An axiomatization of the Banzhaf value. International Journal of Game Theory, 17:89-99.

Macho-Stadler, I., Pérez-Castrillo, D., and Wettstein, D. (2007). Sharing the surplus: an extension of the Shapley value for environments with externalities. Journal of Economic Theory, 135:339-356.

Malawski, M. (2002). Equal treatment, ssymmetry and Banzhaf value axiomatizations. International Journal of Game Theory, 31:47-67.

May, K. O. (1952). A set of independent necessary and sufficient conditions for simple majority decision. Econometrica, pages 680-684.

Myerson, R. (1977). Values of games in partition function form. International Journal of Game Theory, 6:23-31.

Nowak, A. S. (1997). On an axiomatization of the Banzhaf value without the additivity axiom. International Journal of Game Theory, 26:137-141.

Owen, G. (1975). Multilinear Extensions and the Banzhaf value. Naval Research Logistics Quarterly, pages 741-750.

Penrose, L. (1946). The Elementray Statistics of Majority Voting. Journal of the Royal Statistical Society, 109:53-57.

Pham Do, K. and Norde, H. (2007). The Shapley value for partition function form games. International Game Theory Review, 9:353-360.

Shapley, L. S. and Shubik, M. (1954). A method for evaluating the distribution of power in a committee system. American Political Science Review, 48(03):787-792.

Thrall, R. and Lucas, W. (1963). n-person games in partition function form. Naval Research Logistics Quarterly, 10:281-298. 